\title{
Selective zoning of high harmonic emission using counter-propagating light
}

\author{
J. Peatross, S. Voronov, and I. Prokopovich \\ Dept. of Physics and Astronomy, Brigham Young University, Provo, UT 84602 \\ peat@byu.edu
}

\begin{abstract}
High harmonic production can be dramatically increased by utilizing an interaction region much longer than a coherence length. Counter-propagating light pulses can be used to disrupt the out-of-phase harmonic emission from selected zones in the focus so that the remaining emission builds constructively. Counter-propagating light creates a standing field modulation repeating over a half laser wavelength in which phase cancellations for harmonic emission occur. A simple power-law model is used to demonstrate how such pulses can be designed to counteract geometrical phase mismatches and improve emission for individual harmonics by more than two orders of magnitude.
\end{abstract}

(C)1997 Optical Society of America

OCIS code: (190.4160) Multiharmonic generation

\section{References and links}

1. L'Huillier and Ph. Balcou, "High-Order harmonic Generation in Rare Gases with a 1-ps 1053nm Laser," Phys. Rev. Lett. 70, 774 (1993).

2. Zhou, J. Peatross, M. M. Murnane, H. C. Kapteyn, and I. P. Christov, "Enhanced High-Harmonic Generation Using 25 Femtosecond Laser Pulses," Phys. Rev. Lett. 76, 752 (1996).

3. Ditmire, K. Kulander, J. K. Crane, H. Nguyen, M. D. Perry, "Calculation and Measurement of High-Order Harmonic Energy Yields in Helium," J. Opt. Soc. of Am. B 13, 406.

4. Peatross, J. Zhou, I. Christov, A. Rundquist, M. M. Murnane, H. C. Kapteyn, "High-Order Harmonic Generation with a 25 Femtosecond Laser Pulse," in Proceedings of the NATO Advanced Research Workshop on Super Intense Laser-Atom Physics IV (Moscow, Russia 1995) p. 455.

5. V. T. Platonenko, V. V. Strelkov, G. Ferrante, V. Miceli, E. Fiordilino, "Control of the Spectral Width and Pulse Duration of a Single High-Order Harmonic, " Laser Phys. 6, p. 1164-1167 (1996).

6. Fiordilino and V. Miceli, "Laser Pulse Shape Effects in Harmonic Generation from a Two-Level Atom, " J. Mod. Opt. 41, 1415-1426 (1994).

7. Kohler, V. V. Yakovlev, Jianwei Che, J. L. Krause, M. Messina, K. R. Wilson, N. Schwentner, R. M. Whitnell, and Yijing Yan, "Quantum Control of Wave Packet Evolution with Tailored Femtosecond Pulses," Phys. Rev. Lett. 74, 3360-3363 (1995).

8. L'Huillier, K. J. Schafer, and K. C. Kulander, "Theoretical Aspects of Intense Field Harmonic Generation, "J. Phys. B: At. Mol. Opt. Phys. 24, 3315 (1991).

9. L'Huillier, Ph. Balcou, S. Candel, K. J. Schafer, and K. C. Kulander, "Calculations of High-Order HarmonicGeneration Processes in Xenon at 1064 nm," Phys. Rev. A 46, 2778-2790 (1992).

10. Ph. Balcou and A. L'Huillier, "Phase-Matching Effects in Strong-Field Harmonic Generation," Phys. Rev. A 47, 1447-1459 (1993).

11. Peatross and D. D. Meyerhofer, "The Angular Distribution of High-Order Harmonics Emitted from Rare Gases at Low Density," Phys. Rev. A 51, R906 (1995).

12. Peatross and D. D. Meyerhofer, "Intensity-Dependent Phase Effects in High-Order Harmonic Generation," Phys. Rev. A 52, 3976-3987 (1995).

13. Peatross, M. V. Fedorov, K. C. Kulander, "Intensity-Dependent Phase-Matching Effects in Harmonic Generation," J. Opt. Soc. Am. B 12,863 (1995).

14. Salieres, A. L'Huillier, and M. Lewenstein, "Coherence Control of High-Order Harmonics," Phys. Rev. Lett. 74, 3776 (1995).

15. Wahlstrom, J. Larsson, A. Persson, T. Starczewski, and S. Svanberg, "High-Order Harmonic Generation in Rare Gases with an Intense Short-Pulse Laser," Phys. Rev. A. 48, 4709 (1993).

16. Altucci, T. Starczewski, E. Mevel, C.-G. Wahlstrom, B. Carre, A. L'Huillier, "Influence of Atomic Density in High-Order Harmonic Generation," J. Opt. Soc. Am. B 13, 148 (1996). 
17. Lynga, A. L'Huillier, C.-G. Wahlstrom, "High-Order Harmonic Generation in Molecular Gases," J. Phys. B 29, 3293 (1996).

18. T. Ditmire, J. W. G. Tisch, D. J. Fraser, J. P. Marangos, N. Hay, M. H. R. Hutchinson, T. Donnelly, R. W. Falcone, M. D. Perry, "High-Order Harmonic Generation in Large Molecules and Atomic Clusters," in Conference on Lasers and Electro-Optics, Vol. 9 of 1996 OSA Technical Digest Series (Optical Society of America, Washington, DC, 1996) p. 544.

19. Wahlstrom, S. Borgstrom, J. Larsson, and S.-G. Pettersson, "High-Order Harmonic Generation in LaserProduced Ions Using a Near-Infrared Laser," Phys. Rev. A 51, 585 (1995).

20. Norreys, M. Zepf, S. Moustaizis, A. P. Fews, J. Zhang, P. Lee, M. Bakarezos, C. N. Danson, A. Dyson, P. Gibbon, P. Loukakos, D. Neely, F. N. Walsh, J. S. Wark, A. E. Dangor, "Efficient Extreme UV Harmonics Generated from Picosecond Laser Pulse Interactions with Solid Targets," Phys. Rev. Lett. 76, 1832 (1996).

21. H. M. Milchberg, C. G. Durfee, T. J. McIlrath, "High-Order Frequency Conversion in the Plasma Waveguide," Phys. Rev. Lett. 75, 2494 (1995).

22. Shkolnikov, A. E. Kaplan, and A. Lago, "Phase-Matching Optimization of Large-Scale Nonlinear Frequency Upconversion in Neutral and Ionized Gases," J. Opt. Soc. Am. B 13, 412 (1996).

23. Armstrong, N. Bloembergen, J. Ducuing, and P. S. Pershan, Phys. Rev. 127, 1918 (1962).

24. Birulin, V. T. Platonenko, and V. V. Strelkov, "High-Harmonic Generation in Interfering Waves," JETP 83, 33 (1996).

25. Peatross, J. Chaloupka, and D. D. Meyerhofer, "High-Order Harmonic Generation with an Annular Laser Beam," Opt. Lett. 19, 942 (1994).

\section{Introduction}

When an intense laser pulse is focused into an atomic medium (typically low-density gas), very many orders of harmonic light can be produced (wavelengths less than a hundredth the laser wavelength). ${ }^{1-2}$ The harmonics have become an attractive source for coherent vacuum ultraviolet radiation. However, in many cases the generated harmonics (in particular the highest orders) have so little energy per pulse as to make them difficult to register, and this limits the prospects of their usefulness. The efficiency of converting the laser light into the high-order harmonics under optimal conditions has reached about $10^{-7}(25 \mathrm{~nm} \mathrm{light})^{3}$ and is often much less. Some improvements to the efficiency of converting the laser light into the high-order harmonics can be made by decreasing the laser pulse duration ${ }^{4}$ or by designing laser pulse temporal profiles to enhance the atomic response. ${ }^{5-7}$ The efficiency of converting laser light into high-order harmonics is seriously limited by several macroscopic mechanisms (other than the atomic response itself): 1. Geometrical phase mismatches arise from discrepancies between the diffraction rates for the laser and for individual harmonics; 2. The refractive index for laser light in the generating medium can differ from the refractive index for the harmonics (particularly severe when free electrons are present); 3. The intrinsic phase of harmonic emission can vary spatially via the atomic response to local laser intensity throughout the focus. All together, these phase mismatches can cause strong cancellations as harmonic light emerges from different locations in the laser focus.

L'Huillier et al. have investigated extensively the phase matching issues associated with focal geometry. ${ }^{8-10}$ In response to such investigations, it is typical to confine the interaction region to a geometrical coherence length to avoid destructive phase cancellations. This is usually done by lengthening the laser focus relative to the width of the gas jet or by working outside of the focus. Nevertheless, since only a very small fraction of the laser energy is converted into harmonic radiation, this confinement of the interaction region is disheartening since the laser intensity in near-by regions is not utilized. The discovery of intensity-dependent intrinsic phases ${ }^{11-12}$ initiated investigations into the possibility of playing the intrinsic phases against the geometrical phase mismatches in an effort to improve overall harmonic generation. ${ }^{13-14}$ However, this seems to produce only marginal gains (i.e., a factor of 2). Wahlstrom et al. ${ }^{15-16}$ have investigated the harmonic generation process as a function of atomic density. They found well-defined limitations to the gas density depending on harmonic order and atomic species beyond which index-related phase mismatches overcame the benefit of an increased number of harmonic emitters. The use of 
atomic clusters or ions as a generating medium so far has not lead to significantly increased conversion efficiencies. ${ }^{17-18}$ On the other hand, high-order harmonic generation in plasma interactions on solid targets using extremely high intensities (i.e. $\sim 10^{19} \mathrm{~W} / \mathrm{cm}^{2}$ ) has recently been demonstrated to have conversion efficiencies in the neighborhood of $10^{-6}$, assuming that the radiation is spread over a wide solid angle. ${ }^{20}$ It should also be noted that Milchberg et al. $^{21}$ have proposed using a pre-formed plasma waveguide to improve phase matching.

In the past few years, a basic understanding of the phase-matching for high harmonics generated in a gas has emerged. The limitations are so severe that under current norms it is more appropriate to call it phase mismatch minimization rather than phasematching. In spite of the difficulties, the possibility of finding ways to achieve genuine phase-matching offers large incentive for addressing the problem. A hundred fold or better improvement to the conversion efficiency would make the harmonics a more attractive vacuum ultraviolet source as well as improve the range over which the harmonics can be studied. Shkolnikov et al. ${ }^{22}$ have recently made estimates on possible increases to the harmonic conversion efficiency based on long-understood ideas of quasi phase matching. ${ }^{23}$ In their work, they point out that if the atomic density can be modulated along the laser axis in order to eliminate harmonic emission from regions having destructive phases, the efficiency of harmonic production can be improved by orders of magnitude. This procedure would compensate for geometrical phase mismatches, but it could not deal with index-related phase mismatches, assuming them to be inseparably linked with the density modulation. The detailed manipulation of the atomic density in the focus poses some technical challenges.

In this article, we propose an alternative approach for achieving quasi phase matching. Rather than attempting to modulate spatially either the density of the atomic medium or the density of free electrons, the approach uses counter propagating light to disrupt the phase matching microscopically in specific regions of the focus. We give an analysis of how even relatively weak counter-propagating light can substantially suppress harmonic production. The quasi phase matching is achieved using multiple counterpropagating light pulses which collide with the main generating pulse in the laser focus. The timing and durations of these counter-propagating pulses can be chosen appropriately to frustrate harmonic production in selected zones with undesirable phase. The remaining regions then constructively interfere, and harmonic production is expected to increase approximately quadratically with the number of in-phase regions created.

It should be mentioned that our approach is fundamentally different from other proposed phase-matching methods using dual light sources. In these cases, either differencefrequency mixing 22 or angular tuning of crossing beams ${ }^{24}$ are proposed to allow for phase matching in a positive dispersive medium (i.e., plasma).
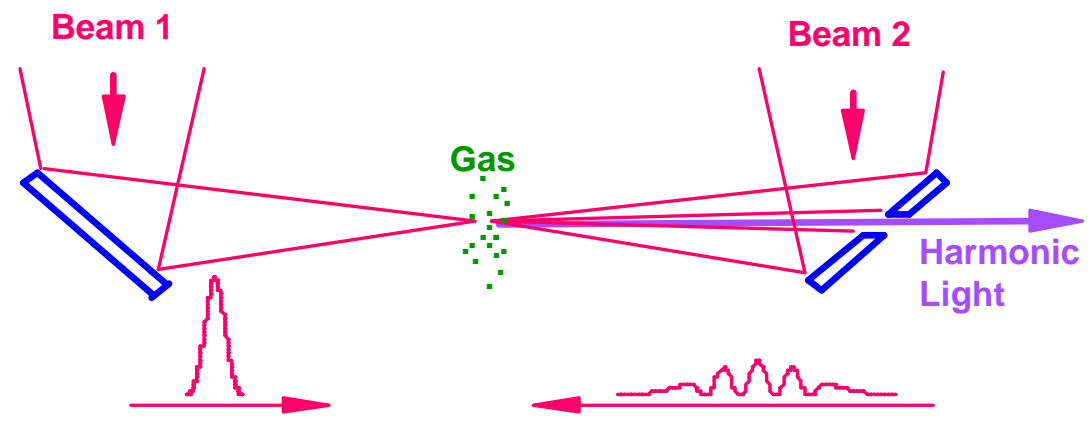

Fig. 1. A mirror with a hole is used to extract high-order harmonics generated in counterpropagating laser beams. 
In Ref. [13], a scheme is proposed for examining intrinsic phases in high-harmonic emission based on the generation of harmonics in the presence of a weak counterpropagating laser beam. Intensity-dependent phases are expected to overcome the extremely poor phase matching in the direction of the weak counter-propagating light and produce measurable albeit faint harmonic signals. It was also pointed out in Ref. [13] that the harmonic emission in the direction of the main pulse is at the same time strongly attenuated (by roughly a factor of ten) owing to a standing interference induced by the weak counterpropagating light. It is this suppression of production in the forward direction that makes counter-propagating pulses an attractive candidate for use in quasi phase-matching.

Figure 1 shows a schematic of the experimental setup which uses a mirror with a hole in it to introduce the counter-propagating light while allowing the harmonics to be observed. $^{25}$ When an annular beam is focused, the center fills in to produce a central peak similar to that of a usual laser focus, surrounded by faint rings which do not contribute to harmonic production. Much of the harmonic energy emerges close to the laser axis so that it can pass inside of the hole. The hole is necessary because of the lack of suitable materials able to transmit vacuum ultraviolet light.

Counter-propagating light disrupts phase matching over a distance of a half laser wave length. This is caused both by a standing phase variation in the crossing beams as well as by the intensity-dependent intrinsic atomic phase responding to the standing intensity fluctuation. In the present work, we will consider only the standing phase variation, although the neglected intensity-dependent phase variation is expected to enhance phase disruption. We will give a microscopic analysis of how counter-propagating light suppresses harmonic production. We will also demonstrate how a tailored series of counter-propagating pulses can be used to overcome geometrical phase mismatches in a Gaussian laser focus.

\section{Microscopic phase disruption in counter-propagating pulses}

Consider two plane wave fields with real amplitudes $E_{1}$ and $E_{2}$ which propagate in opposite directions. We will assume that $\mathrm{E}_{1}$ is the larger of the fields and write their sum as a single field having the form of the stronger plane wave, although with a standing intensity and phase modulation:

$$
E_{1} e^{i(k z-\omega t)}+E_{2} e^{-i(k z+\omega t)}=E_{t}(z) e^{i(k z-\omega t+\phi(z))}
$$

The total amplitude which is stationary and varies spatially can be written as

$$
E_{t}(z)=E_{1} \sqrt{1+\left(\frac{E_{2}}{E_{1}}\right)^{2}+2 \frac{E_{2}}{E_{1}} \cos 2 k z},
$$

and the phase modulation, also stationary, is found to be

$$
\phi(z)=-\tan ^{-1} \frac{\frac{E_{2}}{E_{1}} \sin 2 k z}{1+\frac{E_{2}}{E_{1}} \cos 2 k z} .
$$

In the limit of a very weak counter-propagating field (i.e., $\mathrm{E}_{2}<<\mathrm{E}_{1}$ ), the total field reduces to that of the stronger plane wave (i.e., $\left.E_{t}(z) \rightarrow E_{1} ; \phi(z) \rightarrow 0\right)$. Nevertheless, even a 
relatively weak field $\mathrm{E}_{2}$ can cause significant standing modulations. The fringe visibility for the standing intensity modulation is given by

$$
V=\frac{I_{\max }-I_{\min }}{I_{\max }+I_{\min }}=\frac{2 E_{2} / E_{1}}{1+\left(E_{2} / E_{1}\right)^{2}}
$$

If $\mathrm{E}_{2}$ is only a tenth as strong as $\mathrm{E}_{1}$ (i.e., 100 times less intense), the standing intensity pattern has a fringe visibility of 0.20 . In this case, $I_{\max }$ is $49 \%$ more intense than $I_{\min }$. Fig. 2 shows the standing intensity profile for this case. By intensity, we do not refer to net energy flow (i.e., Poynting vector), but rather to the square of the combined field amplitude (i.e., $\varepsilon_{\mathrm{o}} \mathrm{cE}_{\mathrm{t}}^{2}(\mathrm{z}) / 2$ ). Fig. 2 also shows the standing phase variation described by Eq. (3). Although the phase fluctuates over a total range of only $0.06 \pi$, this translates into a phase disruption of more than $\pi$ for harmonics beyond the $15^{\text {th }}$ order. As seen in Fig. 2, both the standing intensity modulation and the standing phase modulation are periodic over a half laser wavelength.

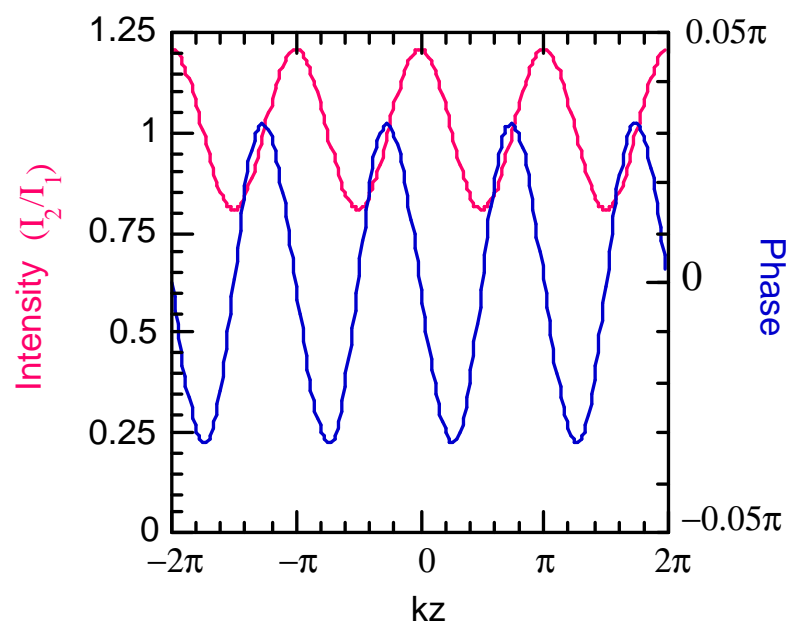

Fig. 2. Standing intensity and phase variations resulting when a plane wave is met by a counterpropagating plane wave one hundredth as intense.

The phase variation within the half wavelength interval can seriously dampen harmonic production. It is useful to perform a microscopic phase-matching calculation over such an interval, say from $-\lambda / 2$ to zero, to find the effective harmonic emission strength to be compared with the usual case of no counter-propagating light (i.e. $E_{2}=0$ ). To perform this calculation, we invoke a simple model: The strength of the $\mathrm{q}^{\text {th }}$ harmonic is assumed to follow the laser field strength raised to the $\mathrm{p}^{\text {th }}$ power. That is, the field emission from individual atoms goes as

$$
\mathrm{E}_{\mathrm{q}}(\mathrm{z}, \mathrm{t}) \sim \mathrm{E}_{\mathrm{t}}^{\mathrm{p}}(\mathrm{z}) \mathrm{e}^{\mathrm{qi}(\mathrm{kz}-\omega \mathrm{t}+\phi(\mathrm{z}))} .
$$

The effective emission from the interval is found by summing the contributions from all participating dipoles to the net field leaving the interval (i.e. at $\mathrm{z}=0$ ). The interval net field at the exiting edge is then proportional to 


$$
\int_{-\lambda / 2}^{0} E_{q}(z, t+z / c) d z \sim e^{-i q \omega t} \int_{-\lambda / 2}^{0} E_{t}^{p}(z) e^{q i \phi(z)} d z .
$$

To obtain a factor proportional to the effective emission from the interval, it is convenient to compare the result of Eq. (6) with $\lambda \mathrm{e}^{-\mathrm{iq \omega} \omega} \mathrm{E}_{1}^{\mathrm{p}} / 2$, the result when $\mathrm{E}_{2}$ is zero. By effective emission, we mean the apparent response of the atoms in the interval as though the phase matching had not been disrupted. We therefore define a microscopic phase-mismatch factor as

$$
\xi \equiv \frac{2}{\lambda E_{1}^{p}} \int_{-\lambda / 2}^{0} E_{t}^{p}(z) e^{q i \phi(z)} d z
$$

Note that $\xi$ is real because $\mathrm{E}_{\mathrm{t}}(\mathrm{z})$ is even and $\phi(\mathrm{z})$ is odd over the interval of integration. Although Eq. (7) is defined in terms of the power law model used in the present work, the definition can be generalized to accommodate other models including results of numerical simulations.

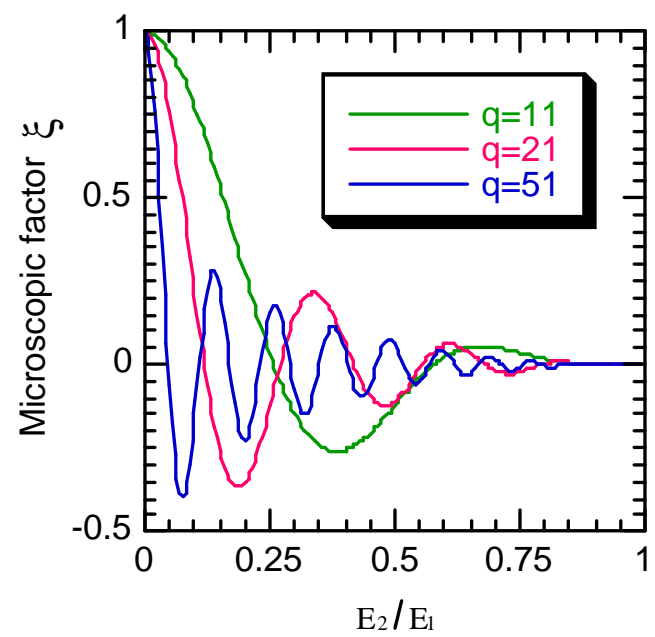

Fig. 3. The effective emission from a microscopic phase-matching interval for the 11 th, 31 st, and 51 st harmonics as a function of relative counter-propagating field strength. The calculation assumes that harmonic emission follows a power law with $\mathrm{p}=5$.

Fig. (3) shows the behavior of the microscopic factor $\xi$ for a variety of harmonic orders as a function of counter-propagating field. In all cases, $p$ is chosen to be 5, but the results are not sensitive to the exact choice for this parameter. As is evident, the emitted harmonics are significantly degraded even with a relatively weak counter-propagating field. For harmonic orders in the twenties or higher, a counter-propagating field strength of only one tenth the main field is sufficient to seriously disrupt the harmonic emission. This means that to realize this situation experimentally, counter-propagating pulses need only have one hundredth the intensity of the main pulse. As is also seen in Fig. 3, the microscopic factor is zero only at a few specific counter-propagating field strengths. Nevertheless, in the cases where the microscopic factor goes negative, the emission is out of phase by $\pi$. Since the reason for suppressing the harmonic production is to eliminate emission having the incorrect 
phase, the $\pi$ phase change is actually more beneficial than if the microscopic factor simply goes to zero. It should be noted that the decrease in the microscopic factor with counterpropagating field strength is entirely due to the functional form of $\phi(\mathrm{z})$; if $\phi(\mathrm{z})$ is arbitrarily held constant, the result of Eq. (7) would continually increase above one.

\section{Geometrical phase mismatches}

The phase matching (mismatch) calculation for harmonics produced in a Gaussian focus under the assumption of perturbation theory (i.e., $\mathrm{E}_{\mathrm{q}} \sim \mathrm{E}_{\mathrm{L}}^{\mathrm{q}}$ ) can be reduced to a onedimensional integral which is well known. ${ }^{8}$ Similarly, the phase matching calculation for the arbitrary power-law model used in the present work (i.e., $\mathrm{E}_{\mathrm{q}} \sim \mathrm{E}_{\mathrm{L}}^{\mathrm{p}}$ ) can be reduced to a one-dimensional integral. ${ }^{12}$ The integral which describes the field of individual harmonics at a screen far from the laser focus is

$\mathrm{E}_{\mathrm{q}}(\theta, \mathrm{T}) \sim \mathrm{E}_{1}^{\mathrm{p}}(\mathrm{T}) \int \mathrm{dz} \frac{\mathrm{N}(\mathrm{z}) \xi(\mathrm{T}, \mathrm{z}) \eta \mathrm{e}^{-\frac{\mathrm{q}^{2} \eta^{2}}{\mathrm{p}}\left(2 \mathrm{f}^{\#} \theta\right)^{2}}}{\left(1+\mathrm{z}^{2} / \mathrm{z}_{\mathrm{o}}^{2}\right)^{(\mathrm{p}-1) / 2}} \mathrm{e}^{-i \tan ^{-1} z \mathrm{z}_{\mathrm{o}}+\mathrm{i} \tan ^{-1} \frac{\mathrm{qz}}{\mathrm{p} z_{\mathrm{o}}}+\mathrm{iq}\left(2 \mathrm{f}^{\#} \theta\right)^{2}\left[1-\frac{\mathrm{q}^{2} \eta^{2}}{\mathrm{p}^{2}}\right] \frac{\mathrm{z}}{\mathrm{z}_{\mathrm{o}}}}$

where $\eta \equiv \sqrt{\left(1+z^{2} / z_{o}^{2}\right) /\left(1+q^{2} z^{2} / p^{2} z_{o}^{2}\right)} . \quad N(z)$ is the density of the atomic medium, $z_{o}$ is the laser Rayleigh range, and $\theta$ is the angle from the axis to a point on the screen. Note that $2 \mathrm{f}^{\#} \theta$ is a parameter which compares the angle of the harmonic emission to that of the laser beam. We have included the microscopic phase-mismatch factor $\xi(\mathrm{T}, \mathrm{z})$ which is identically one in the absence of counter-propagating pulses. $\mathrm{T}$ is a time parameter that refers to a specific point on the temporal envelope of the generating pulse which moves through the focus. In Eq. (8), we have neglected proportionality factors since we are interested only in changes in harmonic production when counter-propagating pulses are used.

The use of the power-law model rather than perturbation theory is significant to the analysis of geometrical phase matching because it allows sizable harmonic production to take place through a longer depth in the focus. This is more realistic than the perturbation model since high-order harmonic generation is known to increase with the laser field at a rate much less than perturbation theory would suggest. Nevertheless, the power law model misses much of the physics associated with realistic atoms and is used here only for its convenience to illustrate geometrical phase mismatch.

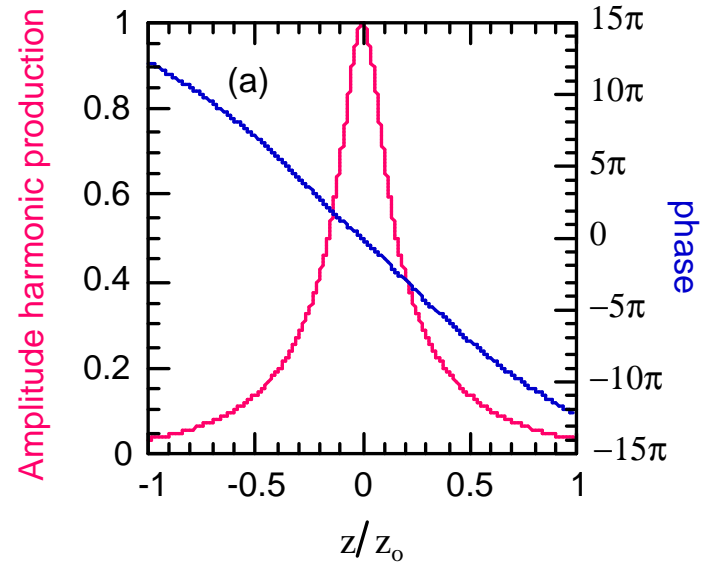

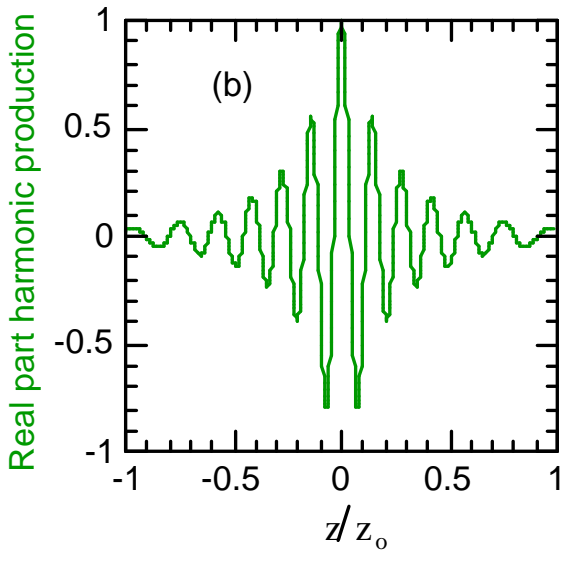

\#2247 - \$10.00 US

(C) 1997 OSA
Received August 1, 1997; Revised August 27, 1997 1 September 1997 / Vol. 1, No. 5 / OPTICS EXPRESS 120 
Fig. 4. (a) Amplitude and phase of the 51st harmonic field arriving simultaneously in the center of a distant screen as a function of emission position (normalized by the Rayleigh range). The graph shows the field contributions associated with a particular point on the pulse temporal envelope as it travels through the focus. The net field for that instant is found by summing the contributions as in Eq. (8). (b) Real part of the field

Fig. 4(a) shows the amplitude and phase of harmonic contributions as a function of point of origin in the laser focus in the absence of a counter-propagating field. This is done for the $51^{\text {st }}$ harmonic with $\mathrm{p}$ chosen to be 5 . The calculation is for that light which hits the screen on axis (i.e., the figure shows the integrand of Eq. (8) with $\theta=0, \mathrm{~N}=1$, and $\xi=1$ ). The phase changes by about $25 \pi$ over the range $-z_{0}<z<z_{0}$. Fig. 4(b) shows the real part of the emission to emphasize the strong effect of the varying phase. The curve in Fig. 4(b) may be interpreted as the electric field contributions from different positions in the focus arriving simultaneously and interfering at the center of the distant screen. Since these contributions arrive at the screen together, it is apparent that they are produced at different laboratory times as a single temporal point $\mathrm{T}$ of the laser pulse moves through the focus. When the contributions are summed (i.e., the integral of Eq. (8) is taken), the strongly varying phase takes a serious toll on the overall harmonic emission. To counteract this effect, counter-propagating pulses can be chosen with appropriate timing and durations so that the microscopic factor $\xi(\mathrm{z}, \mathrm{T})$ tends towards zero in regions with undesirable phase. Alternatively, the gas distribution can be restricted to a coherence length (phase interval of $\pi$ ) to minimize phase cancellations, as is the usual practice, but this makes only limited use of the focal region.

It may be argued that geometrical phase mismatches can be reduced with less trouble by generating harmonics outside of the focus where the laser phase varies less rapidly along the z-axis. However, in order to maintain the intensity necessary for good harmonic production, this requires a shorter depth of focus (assuming fixed laser energy). A decrease in the focal depth increases the rate of axial phase variation, and this cancels the benefit of working outside of the focus. The coherence length for harmonic production is never larger than its value at the focal center as long as the f-number is chosen to make the intensity appropriate at the location of generation. An increase in laser energy does not alter this relationship, since it means simply that a larger f-number can be utilized. Thus, improvements to the phase matching in the vicinity of the focus represent genuine gains in harmonic production. It should be conceded, however, that for very energetic laser systems, it may be more practical to work outside of the laser focus in order to avoid huge f-numbers while fully utilizing available laser energy.

To see why the coherence length at the focal center is as long as it can be, an examination of the phase term $\mathrm{qtan}^{-1} \mathrm{z} / \mathrm{z}_{\mathrm{o}}$ in Eq. (8) is sufficient since it is by far the most rapidly varying phase term. The derivative of this phase term is $q /\left(z_{o}+z^{2} / z_{o}\right)$ which implies a phase change of $\pi$ over an approximate coherence length of $\mathrm{L}_{\mathrm{C}} \cong \pi\left(\mathrm{z}_{\mathrm{o}}+\mathrm{z}^{2} / \mathrm{z}_{\mathrm{o}}\right) / \mathrm{q}$. At an arbitrary position $\mathrm{z}$ away from the focus, the laser intensity drops by the factor $1+z^{2} / z_{0}^{2}$. Thus, in order to satisfy the constraint that the intensity must be the same regardless of position, a new Rayleigh range $z_{0}^{\prime}$ must be chosen depending on the selected position $\mathrm{z}$ such that

$$
\mathrm{z}_{\mathrm{o}} / \mathrm{z}_{\mathrm{o}}^{\prime}=1+\mathrm{z}^{2} / \mathrm{z}_{\mathrm{o}}^{\prime 2}
$$

Under this constraint, the coherence length as a function of position must be written using the new Rayleigh range (i.e., $L_{C} \cong \pi\left(z_{o}^{\prime}+z^{2} / z_{o}^{\prime}\right) / q$ ). A substitution from Eq. (9) simplifies 
the coherence length to $\mathrm{L}_{\mathrm{C}} \cong \pi \mathrm{z}_{\mathrm{o}} / \mathrm{q}$. This result which does not depend on $\mathrm{z}$ implies that the coherence length at the focal center is as long as it is anywhere.

Moreover, away from the focus the harmonic production can tend to zero over a distance shorter than the laser coherence length owing to nonlinearity of the interaction and the decreasing laser intensity associated with the expanding beam. Eq. (8) suggests that the harmonic amplitude decreases with $\mathrm{z}$ as $\left(1+\mathrm{z}^{2} / \mathrm{z}_{\mathrm{o}}^{\prime 2}\right)^{-(\mathrm{p}-1) / 2}$. Again, an estimate using the derivative for harmonic production length yields $L_{P}=z_{o} z_{o}^{\prime} /(p-1) z$, where a substitution from Eq. (9) was again used. From this it is seen that the production length $L_{P}$ will be shorter than the coherence length $L_{C}$ whenever we have $z / z_{o}^{\prime}>q / \pi(p-1)$. This means that the production length can be shorter than the coherence length if the harmonics are generated more than a few Rayleigh ranges from the focus. These results do not contradict reports suggesting improved production slightly outside of the laser focus ${ }^{10,14}$ since the reports refer to experiments made with fixed f-number and higher-than necessary intensity in the focus.

\section{Simulation of harmonic production in a focus with counter-propagating light}

Inasmuch as this analysis uses the results of section 2 which were derived for plane waves, the pulses should be of sufficient duration to enable them to be treated as plane waves over a half laser wavelength. However, as the main laser pulse produces harmonic radiation, the newly created light travels together with the pulse so that harmonics generated by a specific point $\mathrm{T}$ of the laser temporal envelope do not combine with harmonics generated by other points of the envelope (at least not with time separations of more than several harmonic periods as in the case of strong interference). Thus, even though the formulas in Section 2 were derived for counter-propagating plane waves, the main harmonic generating pulse need not be a plane wave. Nevertheless, the use of the plane wave approximation does require the counter-propagating pulse to be of sufficient duration. We have found that Eqs. (2) and (3) hold to within $1 \%$ if the counter-propagating pulse is of duration $100 \mathrm{fs}$ or longer, assuming 800nm light. This corresponds to a distance of $15 \mu \mathrm{m}$ that a point $\mathrm{T}$ on the main pulse travels while experiencing the counter-propagating light. Thus, it appears to be feasible to utilize in excess of ten counter-propagating pulses to dampen harmonic emission from selected zones inside of a $1 \mathrm{~mm}$ region without violating the analytical descriptions above. However, unless the main generating pulse is short compared to the counter-propagating pulses, the selected zones will shift position for different temporal points of the main pulse. However, to the extent that the selected zones are spatially periodic the shifting of the zones will not be important. The quasi plane wave requirement ensures the accuracy of the formulas in section 2, but a non-plane wave is still expected to disrupt harmonic production.

The amplitude of the main pulse envelope has the form $E_{1}(t-z / c)$ while counterpropagating pulses have the form $\mathrm{E}_{2}(\mathrm{t}+\mathrm{z} / \mathrm{c})$. In order to find the relevant ratio between the two crossing fields, we must monitor each temporal point of the main pulse for which $\mathrm{T} \equiv \mathrm{t}-\mathrm{z} / \mathrm{c}$ is constant as it travels through the focus. Thus, the ratio of the main pulse amplitude at point $\mathrm{T}$ to the amplitude of the counter-propagating field throughout the focus is $E_{1}(T) / E_{2}(T+2 z / c)$. With this we can find the microscopic factor $\xi$ as a function of $z$ for each point $\mathrm{T}$ of the main pulse. To do this, we utilize Eqs. (2), (3) and (7). After this preliminary calculation, the integral in Eq. (8) can be computed to find the harmonic emission in the far field.

Fig. 5(a) shows a series of counter-propagating pulses especially tailored to suppress harmonic production in the regions with negative field in Fig. 4(b). The series of pulses was created by blocking portions of a single longer Gaussian. In the laboratory, the counter- 
propagating pulses might be produced using a chirped pulse with selected regions of the spectrum blocked. Depending on which portions of the spectrum are blocked, the duration and timing of the counter-propagating pulses can be controlled. The individual peaks seen in Fig. 5(a) were smoothed to make them appear more experimentally realistic. Although a larger number of peaks would be beneficial, the simulation utilizes seven dips in the counterpropagating light. This number is likely to be experimental feasible. The fact that the intensity is just a hundredth of the main pulse adds to the experimental convenience since the duration might be chosen to be $10 \mathrm{ps}$ (i.e., $1.5 \mathrm{~mm}$ ) or longer. It should be noted that the diversion of laser energy into counter-propagating light lessens harmonic production quadratically (decreasing potential focal area and associated coherence length), so it is desirable to use as little energy as possible to form the counter-propagating peaks. If coherence length is limited by refractive index, then diverted energy would decrease harmonic production only linearly.
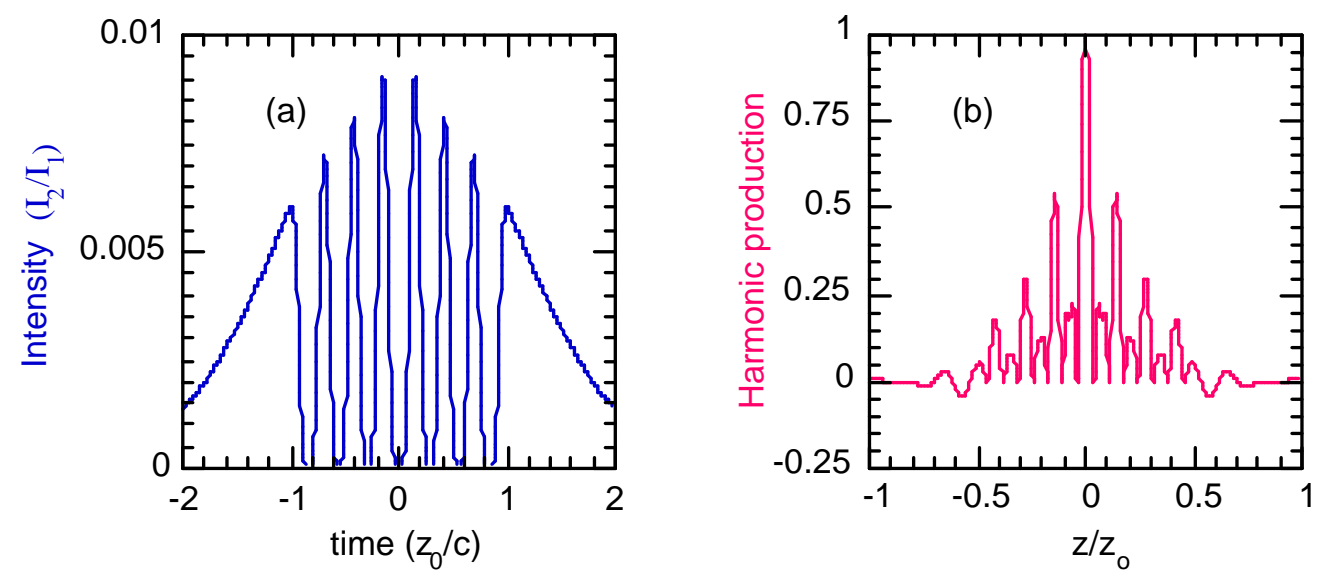

Fig. 5. (a) The relative intensity of counter-propagating pulses tailored to improve emission for the 51st harmonic. (b) The real part of the 51st harmonic field components at the center of a distant screen in the presence of counter-propagating pulses. The graph shows the field contributions associated with a particular point on the pulse temporal envelope as it travels through the focus (position normalized to the Rayleigh range). The net field for that instant is found by summing the contributions as in Eq. (8). Compare with Fig. 4(b).

Fig. 5(b) shows the harmonic production associated with $\mathrm{T}=0$ which must be summed to get the net instantaneous field amplitude on-axis at a distant screen. This is similar to Fig. 4(b) where we have plotted the integrand of Eq. (8) for $\theta=0$, but we have used the microscopic factor $\xi(\mathrm{z}, \mathrm{T}=0)$ computed for the peaks in Fig. 5(a). As can be seen, the out-of-phase portions of the pulse have been removed to a large extent by the counterpropagating light. This produces a very dramatic effect on the integral. Fig. 6(a) shows the results of the integral from $-z_{0}$ to $+z_{0}$ as a function of $\theta$ (blue). The gas density was taken to be uniform over this region, as might be the case for a gas cell. The plot shows intensity (not field) at the screen for the instant associated with $\mathrm{T}=0$ (the laboratory time when the peak of the laser pulse hits the screen). Other values for $\mathrm{T}$ yield similar pictures (assuming a main pulse which is shorter than individual counter-propagating peaks).

The green line in Fig. 6(a) is the result of the integral in the absence of any counterpropagating peaks. The phase cancellations are seen to have a sever effect on harmonic emission. The red line in Fig. 6(a) shows the integral performed in the absence of counterpropagating pulses, but where the density $\mathrm{N}(\mathrm{z})$ is chosen to be a Lorentzian with full-width at half maximum $0.062 \mathrm{z}_{\mathrm{o}}$, a geometrical coherence length. As is evident, restriction of the gas dimension represents a substantial improvement over the case shown by the green line. 
However, the use of counter-propagating light increases the intensity over the narrow jet case by a factor of 40 . Moreover, slightly off axis the intensity has been increased by many orders of magnitude. To compare over all conversion efficiency, it is necessary to sum the intensity over the area on the screen:

$$
\text { Power } \sim \int\left|\mathrm{E}_{\mathrm{q}}(\theta, \mathrm{T})\right|^{2} \theta \mathrm{d} \theta \text {. }
$$

The integration shows for the example in Fig. 6 that the counter-propagating light improves the emitted power relative to the narrow jet case by more than two orders of magnitude. In the example, the counter-propagating pulses were optimized for the on-axis emission, whereas emission at wider angles suffers some phase mismatch. If the counter-propagating pulses are optimized for the emission at wider angles, a conversion efficiency up to three orders of magnitude can be obtained owing to the larger area at the wider angles.
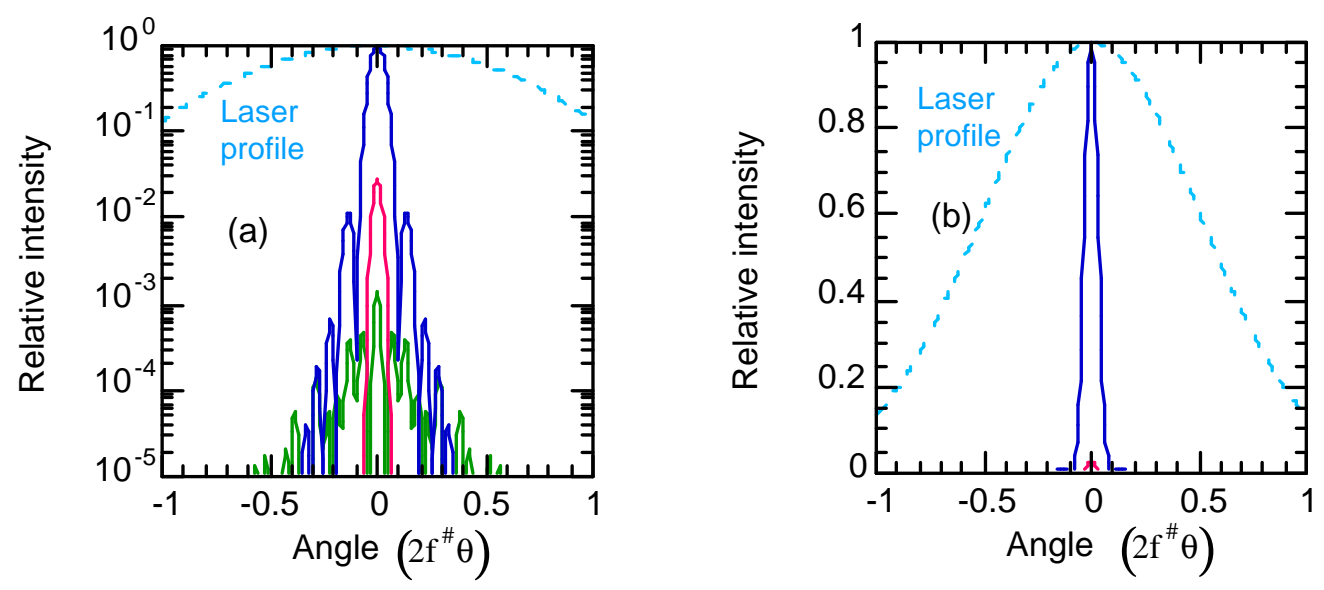

Fig. 6. (a) Relative intensity of the 51 st harmonic at a distant screen as a function of angle, in the presence of the counter-propagating field (blue), in the absence of the counter-propagating field (green), in the absence of the counter-propagating field but with the gas restricted to a coherence length at the focal center (red). The laser profile is depicted for angular comparison (light blue).

(b) The same figure shown with a linear scale.

\section{Summary and discussion}

In this article, we have examined a means of counteracting geometrical phase mismatches in high harmonic generation to enable the use of a longer interaction region. Counterpropagating light appears to offer a practical technique of achieving this by suppressing emission from selected zones having destructive phase. We have shown analytically that even relatively weak counter-propagating light (one hundredth the intensity) can seriously disrupt harmonic emission in the selected zones via a microscopic phase mismatch. Using a simple power-law model to describe harmonic emission, the results of the investigation suggest that it is possible to improve the efficiency of converting laser energy into harmonic radiation by as much as three orders of magnitude.

We have not examined phase mismatches due to free electrons or to the intensitydependent intrinsic phases of the atom. These effects are more difficult to calculate owing to the complicated nature of ionization and emission dynamics. However, it seems likely that counter-propagating light pulses can be applied to counteract these problems in addition to 
the geometrical phase mismatches. The fact that free-electron and intrinsic phase mismatches vary radially in the focus may somewhat frustrate this effort to the extent that the phases at different radii require different sequencing of counter-propagating peaks.

The various phase mismatches are present together as harmonic production takes place, so the optimal timing and spacing of counter-propagating light pulses will ultimately need to be found experimentally. An experimental optimization for the counter-propagating light peaks will automatically take into account phase distortions present in the laser beam. This procedure has the potential to enhance observations of harmonic emission from ions by counteracting the strong disruption to the phase mismatches caused by the free elections. We plan to investigate these issues in upcoming experiments. We acknowledge support for I. Prokopovich from the National Research Council COBASE program. 